Article

\title{
Long-Term Hydrogen Storage-A Case Study Exploring Pathways and Investments
}

\author{
Ciara $\mathrm{O}^{\prime}$ Dwyer $^{1,2}$, Jody Dillon ${ }^{2}(\mathbb{D})$ and Terence $\mathrm{O}^{\prime}$ Donnell ${ }^{1, *}$ \\ 1 School of Electrical and Electronic Engineering, University College Dublin, Belfield, D04 Dublin, Ireland; \\ ciara.odwyer@energyreform.ie \\ 2 Energy Reform, A98 Bray, Ireland; jody.dillon@energyreform.ie \\ * Correspondence: terence.odonnell@ucd.ie
}

Citation: O'Dwyer, C.; Dillon, J.; O'Donnell, T. Long-Term Hydrogen Storage-A Case Study Exploring Pathways and Investments. Energies 2022, 15, 869. https://doi.org/ $10.3390 /$ en15030869

Academic Editors: Zbigniew Leonowicz, Arsalan Najafi and Michał Jasinski

Received: 22 December 2021

Accepted: 19 January 2022

Published: 25 January 2022

Publisher's Note: MDPI stays neutral with regard to jurisdictional claims in published maps and institutional affiliations.

Copyright: (C) 2022 by the authors. Licensee MDPI, Basel, Switzerland. This article is an open access article distributed under the terms and conditions of the Creative Commons Attribution (CC BY) license (https:// creativecommons.org/licenses/by/ $4.0 /$ )

\begin{abstract}
Future low-carbon systems with very high shares of variable renewable generation require complex models to optimise investments and operations, which must capture high degrees of sector coupling, contain high levels of operational and temporal detail, and when considering seasonal storage, be able to optimise both investments and operations over long durations. Standard energy system models often do not adequately address all these issues, which are of great importance when considering investments in emerging energy carriers such as Hydrogen. An advanced energy system model of the Irish power system is built in SpineOpt, which considers a number of future scenarios and explores different pathways to the wide-scale adoption of Hydrogen as a low-carbon energy carrier. The model contains a high degree of both temporal and operational detail, sector coupling, via Hydrogen, is captured and the optimisation of both investments in and operation of large-scale underground Hydrogen storage is demonstrated. The results highlight the importance of model detail and demonstrate how over-investment in renewables occur when the flexibility needs of the system are not adequately captured. The case study shows that in 2030, investments in Hydrogen technologies are limited to scenarios with high fuel and carbon costs, high levels of Hydrogen demand (in this case driven by heating demand facilitated by large Hydrogen networks) or when a breakthrough in electrolyser capital costs and efficiencies occurs. However high levels of investments in Hydrogen technologies occur by 2040 across all considered scenarios. As with the 2030 results, the highest level of investments occur when demand for Hydrogen is high, albeit at a significantly higher level than 2030 with increases in investments of large-scale electrolysers of $538 \%$. Hydrogen fuelled compressed air energy storage emerges as a strong investment candidate across all scenarios, facilitating cost effective power-to-Hydrogen-to-power conversions.
\end{abstract}

Keywords: Hydrogen; renewable energy; investment planning; long-term storage

\section{Introduction}

The decarbonisation of the energy system is a central pillar of climate change mitigation policies and is advancing at a great pace. Electrification of the heating and transport sectors coupled with greatly increased use of renewable generation are generally seen as critical to this decarbonisation effort. The EU has recently proposed to increase its target of energy use from renewable generation to $40 \%$ (from a previous target of $32 \%$ ) as part of a package of measures to put the EU on track for a $55 \%$ reduction in carbon emissions by 2030 compared to 1990 levels, and zero net emissions by 2050 [1]. In Ireland, in $2020,43 \%$ of electricity consumed was generated by renewable sources and a target has been set to increase the share of renewable generation on the grid up to $80 \%$ by 2030 [2]. At these high levels of variable renewable generation, maintaining the supply/demand balance becomes increasingly challenging as balancing challenges occur at vastly different time-scales, from minutes to seasons. Long periods of low renewable generation and high demand can occur at certain periods leading to under supply, while over supply, leading 
to high levels of curtailment become an issue for other periods. While many potential solutions exist, including storages of various durations, flexible generation and demand response, higher degrees of sector coupling can also facilitate more efficient solutions. However, finding optimal solutions is complex, and models must be able to capture the sector coupling, contain sufficient temporal and operational detail and optimise both short duration and seasonal storage over long durations, which is particularly challenging for typical investment models.

Hydrogen is gaining attention as an energy carrier of increasing importance with the potential to provide solutions to supply demand balancing in future high renewable energy systems. It has a wide range of potential applications and pathways [3,4] and can potentially facilitate high levels of sector coupling. "Green Hydrogen", produced by electrolysers using renewable electricity, can be used to avoid curtailment and is a potential fuel source for sectors which are difficult to decarbonise, with applications in industry, and significant potential as a transport fuel, particularly for heavy freight [5]. Hydrogen can also offer zero emissions options in the heating sector, with both the co-generation of heat and electricity via fuel cells, and the deployment of Hydrogen gas boilers emerging as likely candidates to meet future heating needs [6,7]. Hydrogen also has power-to-gas-to-power applications where it has significant potential to provide a clean fuel source for centrally dispatchable generation plants which will still remain important in high renewable systems to provide valuable system services and back up generation when renewable generation is not available. As regards Hydrogen-based generation plant, co-generation of heat and electricity via large-scale fuel cells and Hydrogen fuelled gas turbines are both advancing. However, the efficiency of the combustion process compared to the electro-chemical process is poor [7], and the more efficient co-generation process relies on a local heat demand to take advantage of the increased efficiencies. An alternative option for electricity generation is Hydrogen fuelled compressed air energy storage (CAES), which offers efficiency gains compared to Power-to-Hydrogen-to-power options [8]. While Hydrogen as an energy carrier has a wide range of applications, and offers many advantages as a potentially zero-emission fuel, some of the technologies are still relatively immature and costs are high. It is estimated that billions of euro are required to develop the necessary infrastructure, and support the research and development required to advance emerging technologies and achieve the necessary economies of scale for cost effective generation, storage and transport of the fuel [9]. Despite the enormous costs involved, Hydrogen is receiving very significant interest as the push to reach net zero emissions by 2050 continues, partly due to its potential to store vast amounts of energy at relatively low cost per MWh which is significantly advantageous for seasonal storage [10]. The question then arises, given current cost projections, as to when Hydrogen-based technologies for generation and storage are likely to become commercially advantageous in the energy system. Answering this question requires consideration of the interplay between future projected generation and demand scenarios including increased demand from electrification of heating and transport, and cost projections for Hydrogen-based technologies and their more conventional alternatives. In this work we attempt to answer this question using an optimal investment planning approach which determines the set of technologies which minimize system costs including investment and operational costs.

Modelling the future development of the energy system to achieve decarbonisation goals is challenging for a number of reasons. The potential roles of variable renewables, Hydrogen and long term underground storage give rise to increased interactions across timescales, energy sectors and regions, resulting in a number of modelling challenges. While Hydrogen generation and storage has significant potential value in the future system to facilitate the electrification of sectors such as industry, heating and transport, and can play an important role in system balancing across different time-scales (from minutes to seasons), adequate system models grow in scale and complexity. To effectively capture this potential value, long term investment models must include more operational detail and more energy sectors making them more difficult to solve. Additionally, large shares 
of variable renewable technologies give rise to increased need for flexibility. Adequate temporal and operational detail is therefore essential to capture these flexibility needs and the value flexible technologies can have in meeting these. For example system reserve and inertia which are required to manage short term variations in renewable generation and which should influence longer-term technology investments. In addition, there is a need to optimise long term storage investments and utilisation where energy can be arbitraged across seasons. Long-term investment models thus need to simultaneously allow short term operational issues to influence long term investments while also optimising long term storage investments.This is a challenging proposition because the resulting optimisation models must cover multiple energy sectors over long periods of time at relatively high temporal resolution resulting in a very large problem that is not easily solved.

A number of recent studies have presented energy system investment models, with a focus on Hydrogen technologies. For example, Sgobbi et al. [11] use a TIMES model of the EU28 energy system to assess the role of Hydrogen in a future decarbonised Europe. Yue et al. [12] explore optimal pathways to a $100 \%$ renewable energy system in Ireland, again using a TIMES model and a whole energy system approach. While the TIMES model used for both studies contains a high level of detail in terms of end uses and technologies, just 12 time slices are used. The coarse temporal resolution will not be capable of addressing short-term flexibility needs and curtailment will be underestimated. Optimisation of seasonal storage is also not addressed. Indeed underground Hydrogen storage is not included as an investment option. He et al. [13] develop a generalised modelling framework for co-optimising energy system investments and operations across the power and transport sectors and supply chains of electricity and Hydrogen. Representative weeks with an hourly resolution are used, allowing for the short-term flexibility needs of the system to be addressed. However, once again this approach does not allow for the optimisation of long-duration seasonal storage, and large-scale underground Hydrogen storage is not included as an investment option. Power-to-gas-power offers power systems a high degree of flexibility, but the poor round trip efficiency reduces the viability of such investments. However, Hydrogen fuelled CAES improves the efficiency. To the authors knowledge, no previous studies have included Hydrogen fuelled compressed air energy storage (CAES) as an investment option in low carbon energy system investment models. Standard energy system models are not capable of addressing all the challenges of future system operations with very high shares of variable renewable generation.

This paper presents a case study exploring optimal investments in Hydrogen generation and underground storage alongside other conventional generation and storage technologies in an energy system with a very high share of renewable generation (Ireland). A range of different technologies are considered as investment options. For the conversion of electricity to Hydrogen, electrolysers are considered and for the conversion of Hydrogen to electricity Hydrogen-based Open Cycle Gas Turbines and Hydrogen-based Compressed Air Energy Storage plants are considered. Investment in technologies will of course be influenced by various factors such as technology costs, the costs of alternatives such as conventional fossil fuels, and the costs of renewable generation (as the source of green Hydrogen). The influence of these factors on final investments are explored through the use of a set of scenarios which consider the impact of Hydrogen technology costs and efficiency, Hydrogen demand and fuel and carbon costs, on the uptake and demand for "green Hydrogen" generation and storage investments. A base level of demand for Hydrogen in the transport and industrial sectors is assumed, but as investment is likely to be influenced by potential increased demand in other sectors, one of scenarios specifically looks at how an increased demand for Hydrogen through its use in the heating sector would influence investments. The problem of optimising both investments in and the operation of seasonal storage is dealt with by using enhanced representative periods, which are mapped to equivalent days across the year, the trajectory of the large-scale Hydrogen storage can be optimised as part of the overall solution. The contribution of this paper is the exploration of alternative pathways for "green Hydrogen" investments, examining, through a case 
study, different drivers for investment decisions using an energy system model with high operational and temporal detail. The case study also demonstrates the optimisation of long-term storage investments and operations. The importance of the operational detail and the synergistic impact on multiple investment decisions according to the level of detail included is also highlighted. The results also highlight some of the complementarities and competition which exists between different solutions such as between conventional generation, battery storage and Hydrogen storage and renewable generation. The overall objective of the study is to explore important drivers for the introduction of large-scale Hydrogen investments in an energy system which targets a high levels of variable renewable generation. Although this is explored in the context of a specific case study based on Ireland, the authors believe that the insights gained have relevance to other systems which target very high levels of variable renewable generation.

The remainder of the paper is organised as follows: Section 2 describes the methodology, outlining the scenarios explored, the test system and provides details of the Hydrogen conversion and storage modelling. Section 3 summarises the results of the simulations while Section 4 discusses the implications of these results, along with the limitations of the model and recommendations for future work. Section 5 concludes.

\section{Materials and Methods}

This Section outlines the methodology used to optimise the investment decisions and storage trajectories outlined in Section 3. The objective was to demonstrate alternative pathways for large-scale Hydrogen investments, including long-term storage. An investment model is solved for two study years (2030 and 2040) considering annualised capital costs with an assumed discount rate of $6 \%$. A high level of operational detail facilitates a detailed representation of operational costs, including O\&M costs, start-up costs, fuel costs, taxes and penalties. First, a description of the scenarios explored is provided which focus on future pathways where Hydrogen plays a significant role in the drive towards a net-zero energy system. A description of the test system along with the implementation of the investment model in the SpineOpt tool [14] is provided, along with some more specific modelling details of the Hydrogen conversion processes and long-term storage.

\subsection{Scenarios}

A total of six scenarios are explored for two future years (2030 and 2040). In addition, a further set of simulations are completed for the six scenarios (2030 only) with a lower level of operational detail, in order to explore the impact and highlight the importance of considering a high level of operational detail in investment models exploring the future role of Hydrogen.

The ENTSO-E TYNDP 2020 [15] Global Ambition scenario (GA) was chosen as a base scenario for this work. Wind, solar and load time series, as well as Hydrogen demand and fuel and carbon prices are all based on the TYNDP Global Ambition scenario. Generation capacities for technologies which are not included as investment options in this work (e.g., hydro, waste, biomass) are also taken from this scenario. The remaining five scenarios facilitate the exploration of alternative pathways for Hydrogen adoption and the integration of large-scale Hydrogen storage within future energy systems. The six modelled scenarios are described below:

Global Ambition (GA): The Global Ambition scenario is one of three scenarios considered in ENTSO-E TYNDP 2020 and is in line with COP21 targets. In this scenario, there is a focus on a centralised approach to the energy transition. This scenario has been chosen as the base case for this work.

High Fuel Price (HFP): The objective of this scenario is to explore the impact of fossil fuel prices on investment decisions. In the High Fuel Price scenario, the assumed fuel prices, including for "blue Hydrogen" (i.e., Hydrogen from methane reforming combined with carbon capture and storage) are increased by $30 \%$ from those assumed in the GA scenario. 
Carbon prices are increased to 50/100 EUR / tonne in 2030/2040, respectively, compared to $35 / 80 € /$ tonne in the GA scenario.

Hydrogen Network (HN): This scenario assesses the impact of increased Hydrogen demand on technology investments. Here, Hydrogen is assumed to meet a portion of the country's heating demand, in addition to the predominant demand from transport and industrial Hydrogen assumed in the GA scenario. Reassigning natural gas pipelines is one of many options being considered for the bulk transport of Hydrogen [16]. A portion of the natural gas network is assumed to be reassigned to carry Hydrogen, facilitating a relatively low cost (for the end-user) conversion to Hydrogen space heating. The HN Hydrogen demand timeseries includes space and water heating demand for 100,000 dwellings in 2030, and 500,000 by 2040. It is also assumed that heat pump uptake is reduced when an alternative low carbon heating solution is available to householders, with the electricity demand in the HN scenario updated to reflect this.

Technology Breakthrough (TB): In the Technology Breakthrough scenario, the impact of uncertain electrolyser prices and efficiencies are explored. Large cost reductions for electolysers are anticipated in the coming decades, in addition to improved efficiencies driven by the ongoing research and development and anticipated economies of scale. The TB scenario assumes investment costs at the lower end of projections $[17,18]$ for both 2030 and 2040 (700/300 EUR / kW, respectively, compared to 1000/600 EUR / kW, respectively, assumed for the GA scenario). Modest efficiency improvements are also assumed.

Variable Renewable Energy (VRE): For "green Hydrogen" to compete with other low carbon solutions and to be adopted at scale, wide-scale infrastructure will be required, as well as low cost and efficient electrolysers. Cost effective "green Hydrogen" will also be reliant on low cost renewable energy. In the Variable Renewable Energy scenario, lower investment costs for both wind and solar generation are assumed, exploring the synergistic relationship between low cost renewable generation and Hydrogen generation and storage.

Restricted CAES (RC): Hydrogen fuelled compressed air energy storage offers a flexible potential investment for future high renewables energy systems, providing valuable peaking capacity and energy storage across different time scales, using both the compressed and stored air, and the stored Hydrogen as a fuel source. However, the locating of large-scale CAES plants is geographically restricted, relying on suitable underground storage (e.g., underground salt formations). In the Restricted CAES scenario, limits are imposed for CAES investments, exploring both the impact on alternative generation and storage solutions and the impact on overall Hydrogen investments when this investment option is limited.

\subsection{Test System and Investment Model}

The test system used for this case study is based on the All-Island power system of Ireland. The input data [19], along with the model [20], are both openly available. Existing power plants which are expected to be still operational are included in the base model. Additional capacities are also included for technologies which are not included as investment options, e.g., waste and biomass plant, which have capacities fixed at the levels assumed for the GA scenario. For variable renewable generation, installed capacities in the base model are based on GA levels. However, additional investments are also possible, allowing total installed capacities to increase, depending on the modelled scenario. Table 1 shows the capacities included in the base model (before investments are considered).

Table 2 shows all considered investment options and the capacity considered for each investment decision. Note that for energy storage investments, decisions in increments of $1 \mathrm{MWh}$ are considered, and for renewable generation and batteries, investment decisions are made in increments of $1 \mathrm{MW}$. For plants with more complex efficiency curves, investments are less granular and standard sizing is assumed, with the conventional plant aligning with the ENTSO-E data. With a focus on very large-scale Hydrogen generation, a plant size of $100 \mathrm{MW}$ has been selected for the electrolyser. For the OCGT and CCGT plant, efficiencies and costs are all based on those assumed for the ENTSO-E TYNDP 2020 Global Ambition scenario. Both power and energy capacity investments can be made independently for the 
batteries, with costs based on [21]. CCS is modelled as a post combustion carbon capture and storage unit. The plant is represented in Spine as two separate units with independent investment variables and associated annualised costs. The plant performance, in terms of fuel use, electricity output and emissions is captured for all operating points at an hourly resolution using the user constraint (see Section 2.3). Costs and performance are modelled as per [22] and plant operation is co-optimised as part of the overall problem, in order to minimise costs, with bypassing of the CCS unit possible. More details are provided for the electrolysers and CAES plant in Section 2.3.

Table 1. Starting installed capacities in the base model.

\begin{tabular}{lc}
\hline Plant Type & Installed Capacity (MW) \\
\hline Gas & 2327 \\
Distillate & 324 \\
Biomass \& Waste & 587 \\
Hydro & 238 \\
PHES & 292 \\
Wind (Onshore) & 9607 \\
Wind (Offshore) & 3430 \\
PV & 860 \\
\hline
\end{tabular}

Table 2. Investment options and capacity available for investment.

\begin{tabular}{lc}
\hline Plant Type & Capacity (MW) \\
\hline CCGT & 400 \\
CCGT-CCS & 400 \\
OCGT & 80 \\
H-OCGT & 80 \\
H-CAES & 160 \\
Wind (Onshore) & 1 \\
Wind (Offshore) & 1 \\
PV & 1 \\
Battery (charge/discharge) & 1 \\
Electrolyser & 100 \\
Energy Storage & Capacity (MWh) \\
Battery (energy) & 1 \\
Compressed Air (energy) & 1 \\
Hydrogen (energy) & 1 \\
\hline
\end{tabular}

The investment model is run for 2030 and 2040 (with the 2040 base portfolio updated based on 2030 results and anticipated retirements) using the SpineOpt co-optimised operations and investments model. SpineOpt is an energy system modelling framework, implemented in Julia [20] and developed specifically for detailed operational and planning studies for future energy systems with high shares of variable renewable generation and complex cross-sectoral interactions. SpineOpt's generic structure consisting of nodes, units and connections allows SpineOpt models to be extended easily to include any number of sectors, commodities and energy conversion units. The flexible spatial, temporal and stochastic structures allow the model detail to be carefully tailored for each sector and region of interest, ensuring meaningful results while managing the computational burden. SpineOpt is open source and the complete code and documentation is available online [23].

The SpineOpt modelling framework implements enhanced representative days with ordering and weighting using the SpinePeriods companion model [24] which allows for the reduction of the model size while capturing arbitrage across the full model optimisation horizon. Each period of the model horizon (which can be flexibly defined by the user, e.g., day or week) is mapped to a corresponding representative period. Most problem variables such as unit flows and unit online statuses exist only for the representative periods, 
thus reducing the size of the overall optimisation problem. However, the state variables of long term storage nodes exist for every real (non-representative) interval over the full model horizon. For each real interval, the storage state variables interacts with the other problem variables from the corresponding mapped representative intervals. This allows the state of charge of long term storage to be optimised across the full optimisation horizon and co-optimised with short term operations.

The objective function is shown in Equation (1), which considers investment costs, O\&M costs, start-up costs, fuel costs, taxes and penalties which are associated with the slack variables of the demand balance and reserve constraints. The Mixed Integer Programming (MIP) optimisation is solved using CPLEX 12.9 [25] and an optimality gap of $1 \%$. It should be noted here, that SpineOpt is a flexible modelling framework that allows specification of a wide variety of energy systems in a very flexible way using only nodes, units and connections. The problem formulation in its most general form is presented in the Mathematical Formulation section of the online documentation [23]. Here we present the formulation of the specific case of the model implemented for this work.

$$
\begin{aligned}
\min o b j & =v_{\text {unit_investment_costs }}+v_{\text {storage_investment_costs }} \\
& +v_{\text {fixed_om_costs }}+v_{\text {variable_om_costs }}+v_{\text {fuel_costs }} \\
& +v_{\text {start_up_costs }}+v_{\text {taxes }}+v_{\text {objective_penalties }}
\end{aligned}
$$

Unit and storage investment decision variables $\left(v_{\text {units_invested }}, v_{\text {storages_invested }}\right)$ are included for all units with a defined $p_{\text {unit_investment_cost }}$ and for all nodes with a defined $p_{\text {storage_investment_costs }}$, which are included in this model as annualised investment costs with an assumed discount rate of $6 \%$. The total unit and storage investment costs are shown below in Equation (2) and Equation (3).

$$
\begin{gathered}
v_{\text {unit_investment_costs }}=\sum_{(u, t)} v_{\text {units_invested }}(u, t) \times p_{\text {unit_investment_cost }}(u, t) \\
v_{\text {storage_investment_costs }}=\sum_{(n, t)} v_{\text {storages_invested }}(n, t) \times p_{\text {storage_investment_cost }}(n, t)
\end{gathered}
$$

In SpineOpt, the temporal resolution of energy flows, unit online decisions and investment decisions can all be defined independently and can change by look-ahead time using temporal block objects. The investment temporal block has a resolution of 1 year while the remaining decision variables have a resolution of 1 hour, using 12 weighted representative days generated using SpinePeriods [24]. A further temporal block is used to define the mapping of the non-representative days to a representative day, which allows the trajectory of long-term storages to be considered and the storage investments to be optimised. This will be described in more detail in Section 2.3.

Time series for demand, wind and solar generation are all taken from ENTSO-E TYNDP 2020 Global Ambition scenario, using the 1984 climate year. Total annual Hydrogen demand is also taken from the Global Ambition scenario, which comes predominantly from the industrial and transport sectors. A weekly profile for the transport related Hydrogen demand based on [26] is applied to the annual estimate for Hydrogen transport demand for Ireland from the Global Ambition scenario. Unit constraints include minimum generation levels and minimum up and down times and start-up costs are included. System constraints include an inertia floor and primary and tertiary operating reserve requirements. In addition to being met by generating units, demand can also be met by demand side response (DSM) with an assumed variable operation and maintenance (VOM) cost of $€ 500 / \mathrm{MWh}$. $10 \%$ of the DSM capacity can also provide system operating reserves. In addition, $2150 \mathrm{MW}$ of DC interconnectors are included -1450 MW to GB and $700 \mathrm{MW}$ to France. GB and France are each represented as a single generating unit with a time varying VOM cost, representing the marginal price, with an average value matching the TYNDP 2020 prices. The VOM varies with net load as per the country specific matched 
times series for TYNDP 2020 Global Ambition. This allows the flows on the interconnectors to be approximated and reserve provision is also facilitated. Future work will use a full European model to estimate the country specific marginal prices.

\subsection{Hydrogen Conversion and Storage}

SpineOpt has been designed as a generic energy system modelling framework and it does not assume specific types of energy carriers or sectors. A wide variety of energy systems, technologies and transport physics can be implemented using the fundamental elements of nodes (representing balance, storage and demand), connections (representing transport) and units (representing conversions). Any number of sectors can be included and co-optimised within the model and arbitrary energy conversion units can be added. SpineOpt is a powerful tool when considering a high degree of sector coupling and when modelling emerging technologies, such as electrolysers and Hydrogen-fueled CAES. This Section provides more details of the Hydrogen technologies included in the model. These include electrolysers for the conversion of electricity to Hydrogen, CAES and Hydrogen turbines for electricity generation and both the underground Hydrogen and compressed air storage. Figure 1 shows a simplified version of the Hydrogen node (labelled "H2")as implemented in the SpineOpt model. In the diagram the SpineOpt objects of units are in red and nodes are in purple, while the black lines represent the relationships between the various objects on which the various model parameters are defined. The red arrows indicate the direction of flow. The Hydrogen node has an associated time varying demand, with the time series depending on the year and the scenario. Storage can be added to the node in SpineOpt by giving the node a state, and for existing storage, defining a node state capacity node_state_cap. For nodes with storage investments enabled, node_state_cap represents the storage capacity per storage investment, which is set at $1 \mathrm{MWh}$ in this model. An importer unit (labelled "Importer_H2") allows "blue Hydrogen" to be imported to the Hydrogen node based on cost estimates for the relevant years. "Blue Hydrogen" is assumed to be an important transitional fuel while "green Hydrogen" scales up. As such, the importer capacity is sized to meet the Hydrogen demand in the 2030 GA scenario. Additional Hydrogen demand, including increases assumed for 2040 scenarios, must be met by the generation of "green Hydrogen". The Hydrogen node, and any invested storage capacity at the Hydrogen node, i.e., underground Hydrogen storage, is connected to the electricity node (labelled "ELEC_IE") via three different unit types: electrolysers, Hydrogen CAES and as an alternative, a Hydrogen gas turbine.

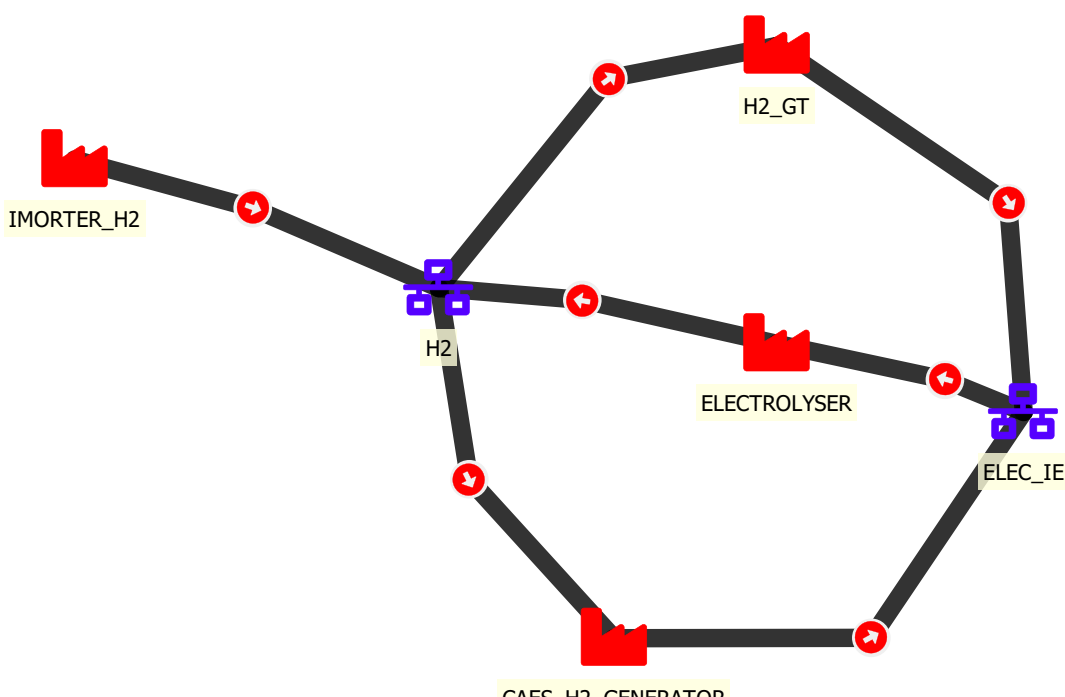

Figure 1. Simplified SpineOpt implementation of the Hydrogen network. 
Units in SpineOpt may have any number of input flows from nodes and any number of output flows to nodes. Arbitrary affine constraints can be defined involving any or all of these flows to represent conversion processes. Electricity can be converted to Hydrogen via the electrolyser units which are included as an investment option. PEM electrolysers are assumed and a detailed operational model is included, with minimum and maximum load levels and efficiency which varies with input electrical energy as outlined in [27]. Here, the electrolyser efficiency curve is approximated using one of SpineOpt's generic conversion constraints. The fix_ratio_out_in_unit_flow constraint in its simplest form allows a linear relationship to be defined between the outgoing flow and the incoming flow from and to a unit (for the elctrolyser the flow of electricity from the electricity node, and the flow of Hydrogen to the Hydrogen node), using the parameter $p_{\text {fix_ratio_out_in_unit_flow. }}$. By including the $p_{\text {fix_units_on_coefficient_out_in }}$ parameter, the varying efficiency of the electrolyser is captured (see Equation (4)). In SpineOpt, more complex efficiency curves can also be represented by defining an array of operating points for the unit, facilitating the decomposition of the flow variable in to multiple segments (i.e., incremental heat rates). Full details are provided in the documentation [23].

$$
\begin{aligned}
& \sum_{\left(u, n, d, t_{\text {out }}\right) \in\left(u, n_{\text {out }}: t t_{-} n o d e, t\right)} v_{\text {unit_flow }}\left(u, n, d, t_{\text {out }}\right) \\
& ==p_{\text {fix_ratio_out_in_unit_flow }}\left(u, n_{\text {out }}, n_{\text {in }}, t\right) \\
& \times \sum_{\left(u, n, d, t_{\text {in }}\right) \in\left(u, n_{\text {in }}, f \text { from_node }, t\right)} v_{\text {unit_flow }}\left(u, n, d, t_{\text {in }}\right) \\
& +p_{f i x \_u n i t s \_o n \_c o e f f i c i e n t \_o u t \_i n}\left(u, n_{\text {out }}, n_{\text {in }}, t\right) \\
& \times \sum_{\left(u, t_{\text {units_on }}\right) \in(u, t)} v_{\text {units_on }}\left(u, t_{\text {units_on }}\right) \\
& \forall\left(u, n_{\text {out }}, n_{\text {in }}\right) \in \text { ind }\left(p_{\text {fix_ratio_out_in_unit_flow }}\right), \\
& \forall t \in \text { time_slices }
\end{aligned}
$$

where $v_{\text {unit_flow }}$ and $v_{\text {units_on }}$ represent the flow and units online variables and the coefficients applied to the variables are the parameters $p_{\text {fix_ratio_out_in_unit_flow }}$ and

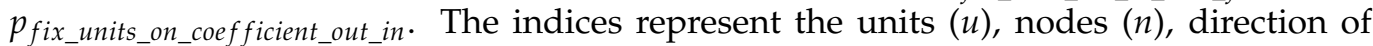
flow $(d)$ and time-slice $(t)$. Equation (4) is applied to all unit, node, node tuples which have a $p_{\text {fix_ratio_out_in_unit_flow }}$ defined-i.e., it can also be applied to conventional generating units, such as the Hydrogen gas turbine, defining the relationship between the flow of Hydrogen to the gas turbine and the flow of electricity to the electricity node.

The CAES plant uses similar generic constraints to describe its operation. However, the CAES plant is slightly more complex, and is modelled as 3 separate units connected to 3 different nodes. Figure 2 shows a simplified implementation of the CAES plant in SpineOpt (for clarity, temporal blocks and stochastic structures are omitted). As per Figure 1, the units are shown in red, and the nodes are in purple. In addition, the yellow symbol represents user defined constraints. The black and grey lines represent various relationships between the model objects, with the black lines also representing flows, with the arrows indicating the direction of flow. Equation (4) is applied to the air compressor unit (CAES_COMPRESSOR in Figure 2) which defines the relationship between the flow of electricity from the electricity node and the flow of air to the compressed air storage node. On the generation side, the CAES plant is modelled as two additional units, CAES_EXPANDER and CAES_H2_GENERATOR in Figure 2, and once again, Equation (4) determines the relationship between the flow of compressed air and Hydrogen from their respective nodes and the flow of electricity from the two units, the combined flow being the power output of the plant. As the two generating units do not operate independently, a further user constraint is applied linking the output of the two units. SpineOpt's user constraint allows the user to define arbitrary linear constraints involving most of the problem variables. Equation (5) shows an instance of the generic user constraint 
with all the relevant parameters, which allows the relationships between the various flows of the CAES generating units to be captured, with the $u c$ index representing the user constraints. In summary, the CAES plant is represented in Spine as three different units, with independent investment variables and associated annualised costs, and a compressed air node, which also has an associated investment variable and annualised investment cost. Equation (4) is used to quantify the efficiency of each unit component of the plant and the user constraint, Equation (5), links the operation of the CAES generating units (air expander and $\mathrm{H} 2$ generator). This methodology allows for a detailed representation of the CAES plant at an hourly resolution in terms of fuel use (both Hydrogen and compressed air) and electricity generation. The state of the compressed air node (i.e., energy content of the compressed air cavern) is also modelled at an hourly resolution and the plant operation is co-optimised as part of the overall investment problem.

$$
\begin{aligned}
& +\sum_{u, n \in u n i t \_n o d e \_u s e r \_c o n s t r a i n t(u c), t} v_{u n i t \_f l o w}(u, n, d, t) \times p_{\text {unit_flow_coefficient }}(u, n, u c, t) \\
& +\sum_{u \in \text { unit_user_constraint }(u c), t} v_{\text {units_on }}(u, t) \times p_{\text {units_on_coefficient }}(u, u c, t)=0 \\
& \forall u c, t \in \text { constraint_user_constraint_indices }
\end{aligned}
$$

As described previously, storage can be added to a node in SpineOpt by giving the node a state, and, for existing storages, defining a node state capacity node_state_cap. When investments in storage capacity at a given node are enabled, node_state_cap represents the storage capacity per storage investment, which is set at $1 \mathrm{MWh}$ in this Model. However, optimising investments in long-term storage is challenging in typical investment models, which rely on reduced temporal representations to maintain tractability for very large problems. Long time horizons can be considered at a low resolution (e.g., one year at a daily resolution) which allows requirements for seasonal storage to be captured [28]. However, such a low resolution does not capture the flexibility needs of systems with high shares of variable generation, for which a high level of temporal detail is essential [29]. While it is possible to capture the systems short term flexibility needs with suitable selected representative periods, in order to simultaneously capture seasonal storage requirements, more advanced methodologies are required [30]. In this work, SpinePeriods [24] generates and orders representative days using an optimisation approach which approximates the annual duration curves [31]. The remaining, non-representative days are each mapped to a representative day, which is used to model the state of charge of a storage node over the full horizon, allowing the consideration of the arbitrage which takes place between the represented days. Thus an energy trajectory of the storage node can be generated and the energy capacity of the storage node optimised. A cyclic condition for the node state is also enforced, which ensures the node state at the end of the optimisation is at least as high as the initial value at the beginning of the optimisation. In SpineOpt, a map containing a representative day for each day in the horizon is included in a third temporal block (along with the investment temporal block, used for the investment decisions, and the representative days which are used for the operational decisions, see Section 2.2) which applies appropriate constraints to the energy level of the long term storage node for each day of the year. The full formulation is described in [30]. 


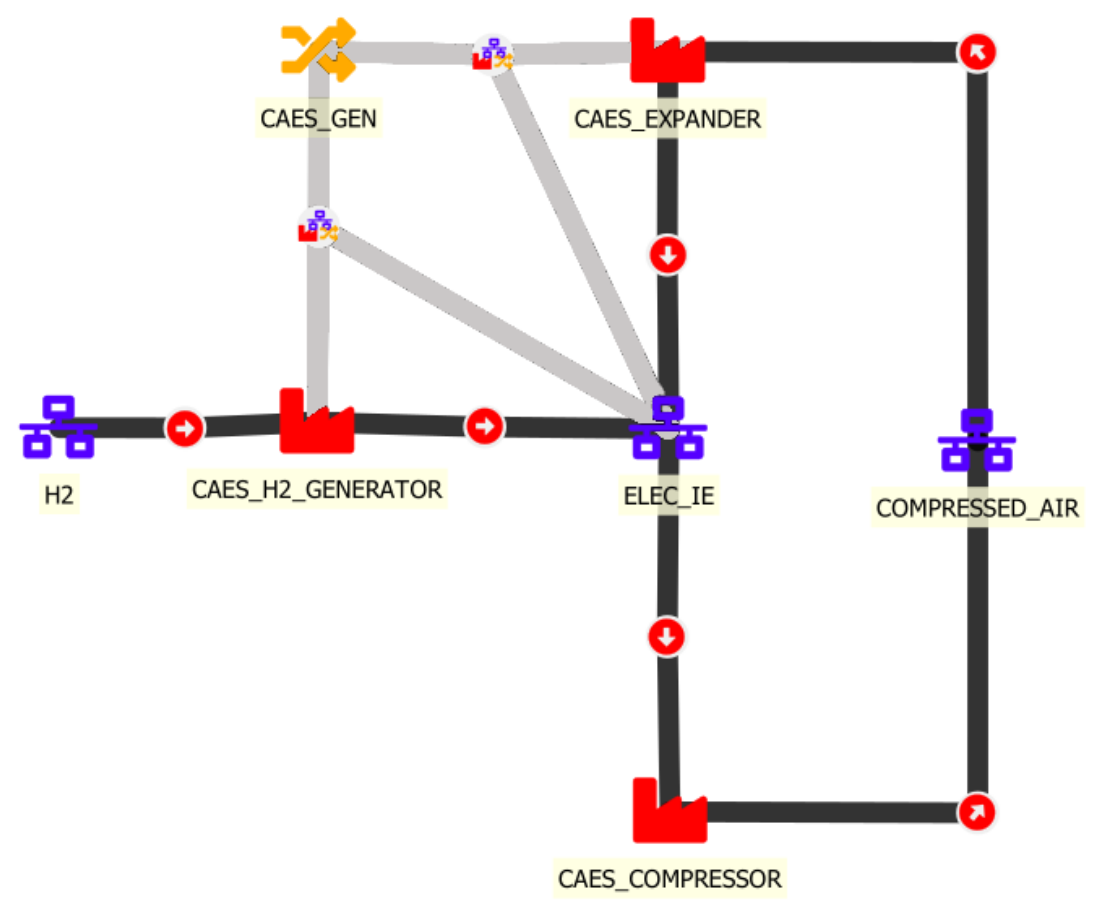

Figure 2. Simplified SpineOpt implementation of the CAES plant.

\section{Results}

The Results of the investment model are shown in this section, firstly for 2030, secondly for 2040 and finally a second set of 2030 results are presented with a lower level of operational detail, highlighting the impact such details have the results of long-term investment models.

\subsection{Results}

Figure 3 shows the capacities of dispatchable generation selected for investment by the 2030 investment models under the different scenarios. A high level of investments in electrolysers occur in the HFP and HN scenarios, with a lower level of investments occurring in the TB scenario triggered by the lower costs and higher efficiencies. However in the other three scenarios no investment in Electrolysers occur. Electrolyser investments are coupled with strong investments in Hydrogen CAES plant, and for the HN scenario, the flexibility and load shifting provided by the electrolysers and CAES suppresses battery investments.

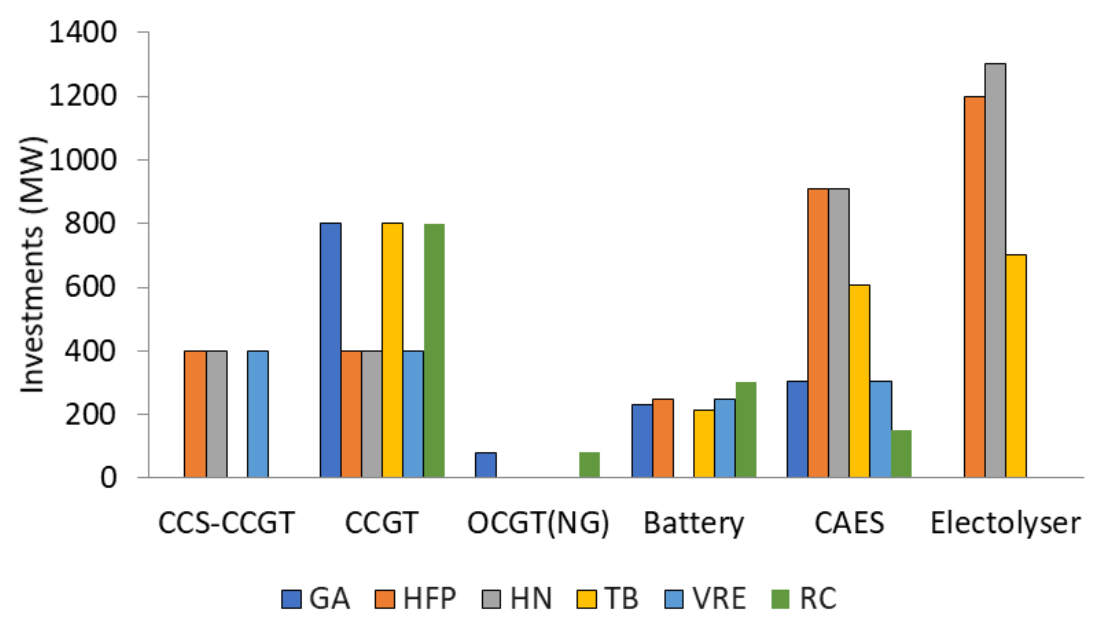

Figure 3. 2030 investments including dispatchable generation capacities and load (electrolysers). OCGT(NG) refers to natural gas powered OCGT. 
In all modelled scenarios, high levels of wind generation are already included (as per the GA scenario). As shown in Figure 4a in 2030, variable renewable investments predominantly occur for PV, despite the relatively poor capacity factor for PV in Ireland. The installed capacity of PV in 2030 is modest (see Table 1) and a combination of favourable capital costs and a relatively high capacity factor in the summer months (when wind availability is lower) triggers a high level of investments. Interestingly, the HN scenario sees the lowest level of PV investments despite the additional Hydrogen demand. However, the additional Hydrogen demand included for this scenario is concentrated in the winter months (heating) when PV availability is poor, although alternative Hydrogen demand assumptions (e.g., higher transport or industry demand) would impact on this result.

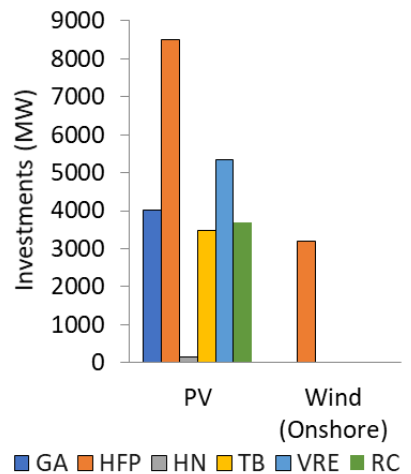

(a)

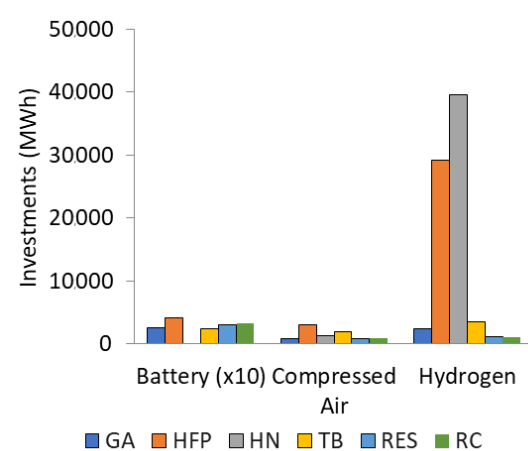

(b)

Figure 4. (a) 2030 investments in variable renewable generation. (b) 2030 investments in energy storage capacity.

The energy storage investment capacities can be seen in Figure 4b. Hydrogen CAES emerges as principal technology selected for short/medium term balancing. However, only the scenarios with highest levels of electrolyser investments (HFP \& HN) see large investments in the underground Hydrogen storage. The stored Hydrogen (primarily generated by the electrolysers) can be used both to meet the defined external Hydrogen demand and also by the dispatchable plant (i.e., CAES).

\subsection{Results}

Figure 5 shows the combined capacities of dispatchable generation selected by the 2030 and 2040 investment models. By 2040, lower costs for electrolysers are assumed as well as increased efficiencies. Fuel costs, and particularly carbon prices are also expected to rise which all favour "green Hydrogen" production. A very high level of investments in electrolysers occur in all scenarios. As with the 2030 scenarios, electrolyser investments are coupled with strong investments in Hydrogen CAES plant, and restricting investments in CAES (RC scenario) has a large impact on the remaining investment options. Investments in electrolysers and renewable generation decrease, and more gas plant is required (both OCGT and CCGT). While a share of "blue Hydrogen" import is still possible (i.e., 2030 demand levels), the imports are largely displaced by "green Hydrogen" production in most scenarios as an economically competitive alternative. 


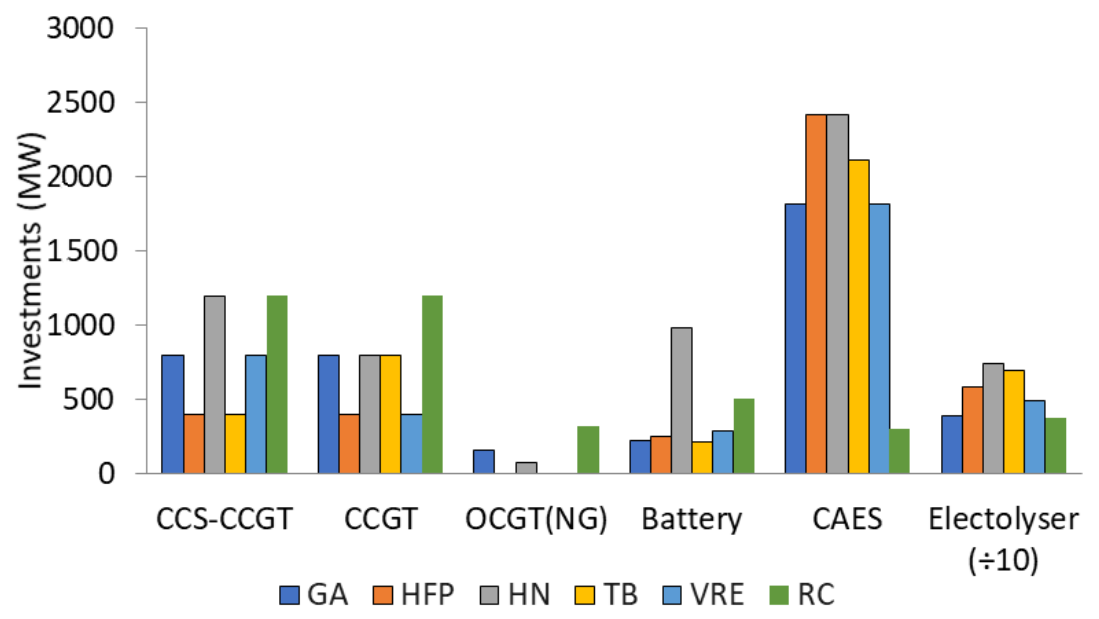

Figure 5. 2040 investments including dispatchable generation capacities and load (electrolysers).

As shown in Figure 6a, as with the 2030 scenarios, PV investments dominate, to complement the already high level of installed wind generation, although wind generation investments also occur for 5 of the 6 scenarios, the exception being the RC scenario. In the 2040 scenarios, with the large capacities of electrolysers, and the high demand for Hydrogen, we now see large investments in the underground Hydrogen storage to facilitate arbitrage across the year for all scenarios (see Figure 6b).

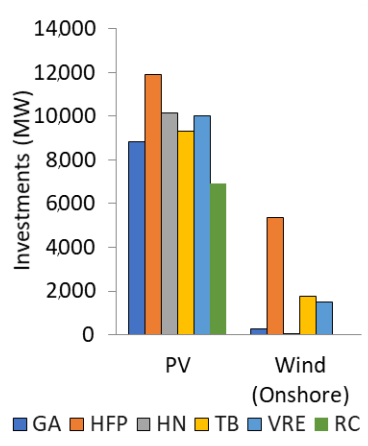

(a)

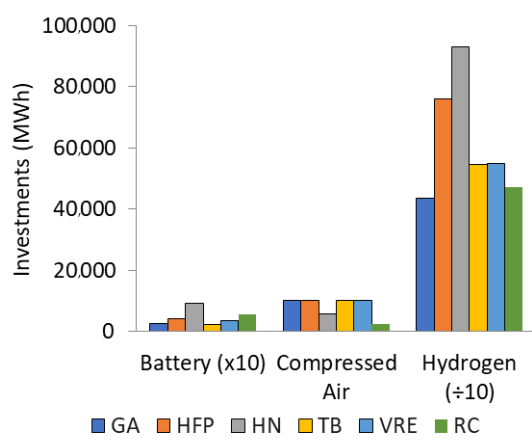

(b)

Figure 6. (a) 2040 investments in variable renewable generation. (b) 2040 investments in energy storage capacity.

Figure 7 shows the state of charge of the underground Hydrogen storage across the full year in 2040 for three of the 2040 scenarios. GA and HFP follow a similar trajectory, albeit with HFP using a larger volume of storage, with a higher reliance on Hydrogen due to the high fuel prices. All three trajectories demonstrate charging when excess renewable generation occurs and discharging when demand for Hydrogen is high and net load is also high (as Hydrogen is used by the CAES plant). Differences in variable renewable investments will drive changes in the shape of the trajectory. The HN trajectory is markedly different from the other two, due to the high Hydrogen demand assumed for heating in the winter months. 


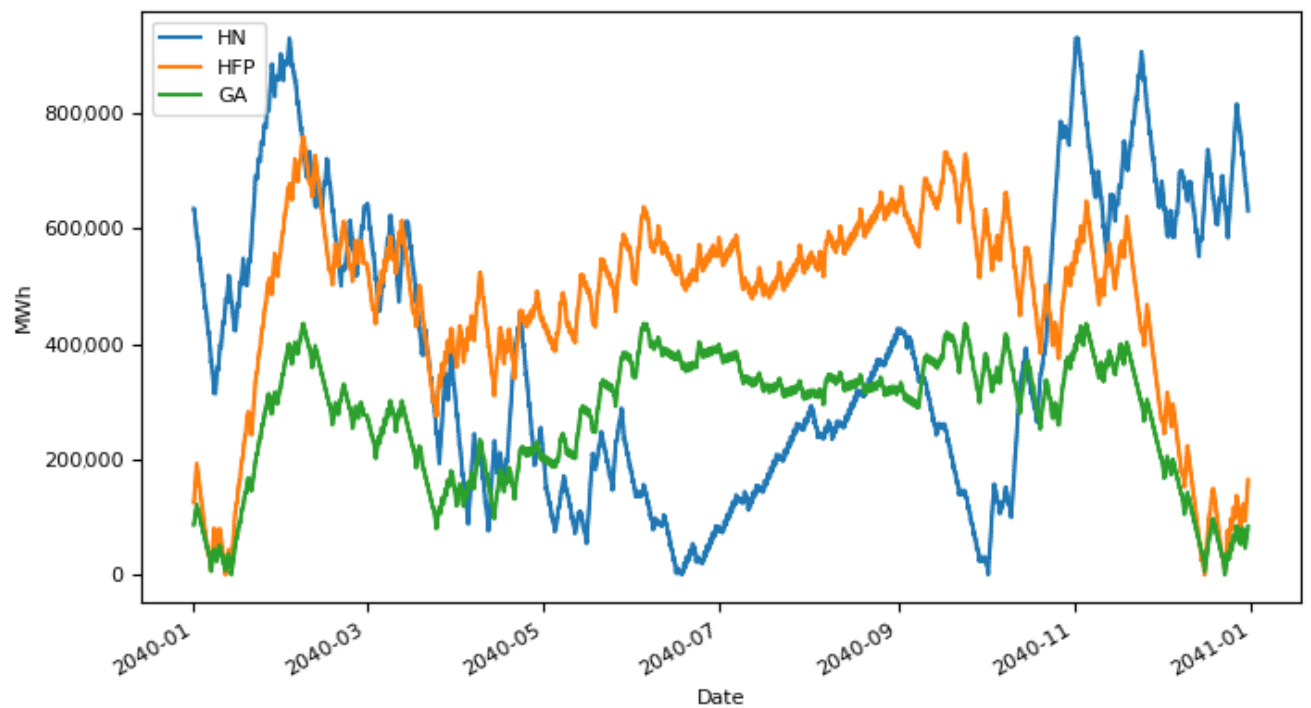

Figure 7. Trajectories of the state of charge of the underground Hydrogen storage for the 2040 scenarios-GA (green), HFP (orange) and HN (blue).

\subsection{Results with Low Level of Operational Detail}

To explore the impact of operational detail on the investment decisions, an additional set of simulations was completed for the 2030 scenarios, without considering either reserve requirements or the inertia floor. Figures 8 and 9 show the investment decisions for these runs. Compared to the original 2030 results, variable renewable generation is consistently over invested in. Neglecting reserve requirements leads to an underestimation of curtailment levels and renewable integration costs. Conventional plants, particularly the larger CCGT, play an important role in meeting the systems inertia requirements. When this constraint is neglected, lower investment levels occur for conventional dispatchable plants, particularly the CCGT (with or without CCS). Despite the higher levels of of renewable generation, as curtailment is underestimated, additional electrolyser investments do not result. Large investments in Hydrogen storage occur for the same two scenarios (HFP, $\mathrm{HN})$, but at significantly higher levels. These differences highlight the importance of cooptimising short term operations with long term investments and storage operation for planning the future energy system, with the ability to include sufficient detail and also the ability to capture the interactions between the different sectors which are becoming increasingly integrated.

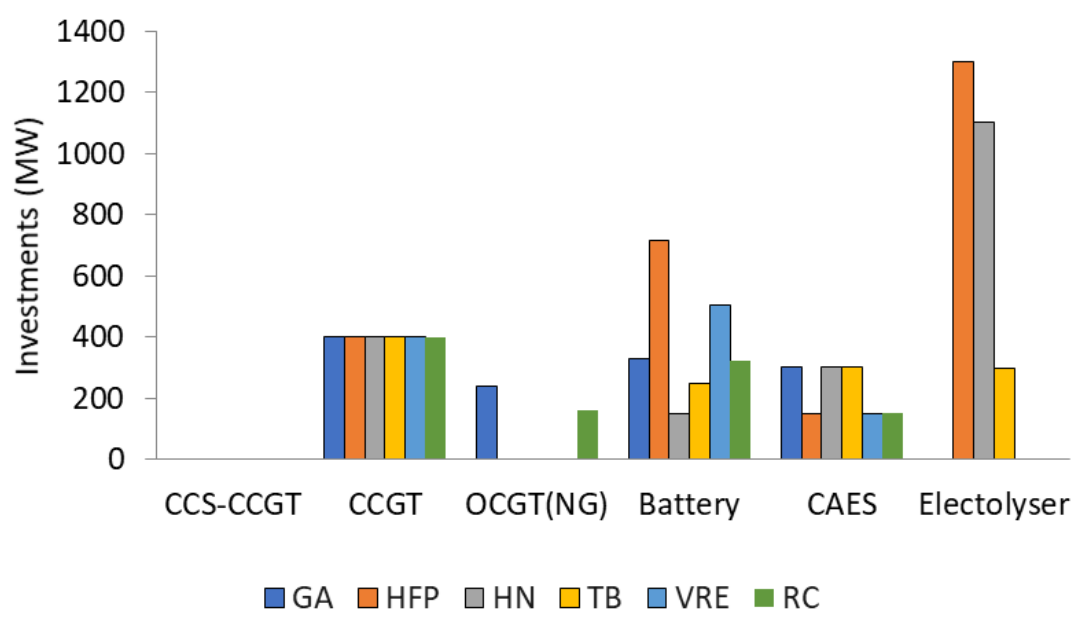

Figure 8. 2030 investments including dispatchable generation capacities and load (electrolysers). 


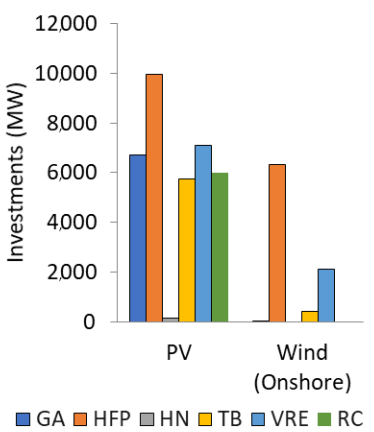

(a)

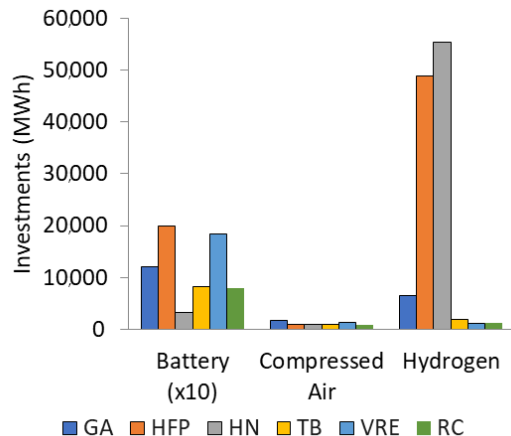

(b)

Figure 9. (a) 2030 investments in variable renewable generation with low operational detail. (b) 2030 investments in energy storage capacity with low operational detail.

\section{Discussion}

Important insights can be gained from the results of this work regarding the potential for the future transition to a Hydrogen economy. "Green Hydrogen" is seen to play a limited role in the 2030 results. In 3 of the 6 scenarios, no investments in large-scale electrolysers occur, and a maximum investment of $1300 \mathrm{MW}$ seen for the remaining 3 scenarios. Limits on "blue Hydrogen" import trigger electrolyser investments in the HN scenario, driven by the increased Hydrogen demand, which represents "green Hydrogen" adoption under a climate of strong policy measures, i.e., strong demand for Hydrogen coupled with limits on "blue Hydrogen" production.Limits on the role of "blue Hydrogen" ties in with the vision of its use as a stepping stone to the wide-scale adoption of Hydrogen as an energy carrier, facilitating increasing demands as "green Hydrogen" scales up and becomes more economically viable. A large uptake of electrolysers is seen for the HFP scenario with investments of $1200 \mathrm{MW}$ occurring in 2030. High fuel prices coupled with a high carbon price triggers a large increase in "green Hydrogen" production. This result highlights the crucial role that carbon prices play in promoting the adoption of costly alternative low-carbon energy solutions. Capital costs of both electroysers and renewable generation influence the uptake in Hydrogen production. While in 2030, capital cost reductions in the VRE scenario are not sufficient to trigger green Hydrogen production, electrolyser investments of $700 \mathrm{MW}$ are seen for the TB scenario. By 2040 the synergistic relationship between variable renewable generation and electrolysers can be seen, with reduced capital costs for either triggering increased investments in both. Indeed, 2040 scenarios provide a much more favourable climate for Hydrogen investments. Again, the HN scenario prompts the highest level of investments with a $634 \%$ increase in electrolyser investments compared to 2030. By 2040 high levels of electrolyser investments are seen across all scenarios, including the base GA scenario, which still sees $60 \%$ of the investments in the HN scenario

The results presented in this paper provide insights into some of the drivers of "green Hydrogen" investments, with the representation of the long-term trajectory of the seasonal storage within the investment model forming an essential part of the optimisation. However, further advances are still required in long-term storage optimisation. Compromises need to be made between model detail and model accuracy, and the selection of an appropriate level of temporal and operational detail is highly system specific [28,30]. Decomposition techniques could be used to facilitate increases levels of operational and temporal detail.

Hydrogen gas turbines are not selected as investments in any of the considered scenarios. The round trip efficiency for power-to-Hydrogen-to-power with a gas turbine used for generation mean the losses are too high for this option to be favoured. An alternative Hydrogen fuelled power generation option is fuel cells which can achieve greater efficiencies when used for the co-generation of heat and electricity. Regions with high levels of district heating anticipate a significant role for fuel cells in power generation. As district 
heating is very limited in Ireland, and due to the large costs and disruptive nature of the addition of district heating, fuel cells were not considered in the six scenarios. Fuel cell uptake in the industrial sector is assumed, represented by the modelled Hydrogen demand. CAES is widely selected in each of the scenarios while, due to the unfavourable economics, Hydrogen turbines saw little investment. Due to geographic limitations, CAES may not be an option in all cases and alternative Hydrogen generation technologies should also be considered in future work.

\section{Conclusions}

Future low-carbon energy systems require radical changes to the structure and operation of the system. Planning for such a future requires complex modelling solutions which must be capable of adequately capturing a high degree of sector coupling at high levels of operational and temporal detail. Insufficient levels of model detail leads to misleading solutions, for example the under estimation of renewables integration costs and curtailment levels, and subsequently over investments in renewable generation. Hydrogen technologies can provide solutions on both the supply and demand side. However it is essential that these investments are not considered in isolation. Hydrogen electrolysers and generators such Hydrogen fuelled CAES can have a synergistic impact on renewable generation investments where investments in one would not be economically viable without the other. Seasonal storage, such as underground Hydrogen storage, can play a vital role in the long-term balancing of the energy system. However, it is difficult to optimise as part of a standard investment model. New methodologies are emerging which allow both investments in and operation of seasonal storage to be optimised, such as using ordered representative periods, mapped to equivalent periods across the year.

The case study explored in this paper demonstrates the use of an energy system model with a high level of sector coupling through the extensive use of Hydrogen as an energy carrier. A high level of operational and temporal detail are included and a methodology which optimises long-term storage is applied. The results show a large role for Hydrogen technologies in Ireland by 2040. The role of Hydrogen in 2030 is more limited, but moderate levels of investment in both electrolysers and Hydrogen fuelled generation (CAES) can still be seen in 2030, when for example, fuel and carbon prices are high, or high levels of Hydrogen demand occur, in this case study prompted by Hydrogen demand in the heating sector. Indeed, high investments in Hydrogen fuelled CAES are seen across all scenarios in this case study, which facilitates increased round trip efficiencies for power-to-Hydrogen-to-power.

Future work will use benders decomposition to iterate between the master investment problem and the operations sub-problem, allowing additional operational and temporal detail to be included, while maintaining tractability. Benders decomposition has already been implemented in SpineOpt, and work on including the long term storage trajectory is progressing. In addition, in future work, alternative generation technologies, including fuel cells will be considered, as well as a more detailed representation of Hydrogen end-uses and alternative storage solutions. A more thorough exploration of alternative pathways to a zero carbon future will also be completed, including different levels of electrification and biomass utilisation.

Author Contributions: Conceptualization, C.O. and J.D.; methodology, C.O. and J.D.; validation, J.D.; formal analysis, C.O.; investigation, C.O. and J.D.; data curation, C.O. and J.D.; writing-original draft preparation, C.O.; writing-review and editing, C.O., J.D. and T.O.; visualization, C.O.; supervision, T.O.; project administration, T.O.; funding acquisition, T.O. All authors have read and agreed to the published version of the manuscript.

Funding: This project has received funding from the European Union's Horizon 2020 research and innovation programme under grant agreement No. 774629.

Institutional Review Board Statement: Not applicable.

Informed Consent Statement: Not applicable. 
Data Availability Statement: The data presented in this study are openly available in: https:// github.com/Spine-project/spine-cs-c3, accessed on 21 January 2022.

Conflicts of Interest: The authors declare no conflict of interest.

$\begin{array}{ll}\text { Abbreviations } \\ \text { The following abbreviations are used in this manuscript: } \\ \text { CAES } & \text { Compressed Air Energy Storage } \\ \text { CCGT } & \text { Closed Cycle Gas Turbine } \\ \text { CCS } & \text { Carbon Capture and Storage } \\ \text { CCGT-CCS } & \text { Closed Cycle Gas Turbine with Carbon Capture and Storage } \\ \text { DSM } & \text { Demand Side Management } \\ \text { ENTSO-E } & \text { European association for the cooperation of transmission system operators (TSOs) } \\ \text { GA } & \text { for electricity } \\ \text { H-CAES } & \text { Global Ambition scenario } \\ \text { H-OCGT } & \text { Hydrogen Compressed Air Energy Storage } \\ \text { HFP } & \text { High Fuel Price scenario } \\ \text { HN } & \text { Hydrogen Network scenario } \\ \text { MIP } & \text { Mixed Integer Programming } \\ \text { OCGT } & \text { Open Cycle Gas Turbine } \\ \text { O\&M } & \text { Operation and Maintenance } \\ \text { RC } & \text { Restricted CAES scenario } \\ \text { TB } & \text { Technology Breakthrough scenario } \\ \text { TYNDP } & \text { Ten Year Network Development Plan } \\ \text { VRE } & \text { Variable Renewable Energy scenario } \\ \text { VOM } & \text { Variable Operation and Maintenance } \\ & \end{array}$

\section{References}

1. European Commission. Proposal for a Directive of the European Parliament and of the Council Amending Directive (EU) 2018/2001 and Directive 98/70/EC as Regards the Promotion of Energy from Renewable Sources, and Repealing Council Directive (EU) 2015/65; Technical Report; European Commission: Brussels, Belgium, 2021.

2. Department of the Environment. Climate Action Plan 2021. Available online: https://www.gov.ie/en/publication/6223eclimate-action-plan-2021/ (accessed on 12 November 2021).

3. Evangelopoulou, S.; Vita, A.D.; Zazias, G.; Capros, P. Energy System Modelling of Carbon-Neutral Hydrogen as an Enabler of Sectoral Integration within a Decarbonization Pathway. Energies 2019, 12, 2551. [CrossRef]

4. Walker, S.; Al-zakwani, S.; Maroufmashat, A.; Fowler, M.; Elkamel, A. Multi-Criteria Examination of Power-to-Gas Pathways under Stochastic Preferences. Energies 2020, 13, 3151. [CrossRef]

5. Moriarty, P.; Honnery, D. Prospects for Hydrogen as a transport fuel. Int. J. Hydrogen Energy 2019, 44, 16029-16037. [CrossRef]

6. Baldino, C.; Searle, S.; Zhou, Y.; Christensen, A. Hydrogen for heating? Decarbonization Options for Households in the United Kingdom in 2050; Technical Report December; The International Council on Clean Transportation: Washington, DC, USA, 2020.

7. Staffell, I.; Scamman, D.; Velazquez Abad, A.; Balcombe, P.; Dodds, P.E.; Ekins, P.; Shah, N.; Ward, K.R. The role of Hydrogen and fuel cells in the global energy system. Energy Environ. Sci. 2019, 12, 463-491. [CrossRef]

8. Bartela, Ł. A hybrid energy storage system using compressed air and Hydrogen as the energy carrier. Energy 2020, 196, 117088. [CrossRef]

9. Erbach, G.; Jensen, L. BRIEFING Towards Climate Neutrality; Technical Report April; European Union: Brussels, Belgium, 2021.

10. Mongird, K.; Viswanathan, V.; Alam, J.; Vartanian, C.; Sprenkle, V.; Baxter, R. 2020 Grid Energy Storage Technology Cost and Performance Assessment; Technical Report December; Pacific Northwest National Laboratory: Richland, WA, USA, 2020.

11. Sgobbi, A.; Nijs, W.; Miglio, R.D.; Chiodi, A.; Gargiulo, M.; Thiel, C. How far away is Hydrogen? Its role in the medium and long-term decarbonisation of the European energy system. Int. J. Hydrogen Energy 2016, 41, 19-35. [CrossRef]

12. Yue, X.; Patankar, N.; Decarolis, J.; Chiodi, A.; Rogan, F.; Deane, J.P.; O'Gallachoir, B. Least cost energy system pathways towards 100\% renewable energy in Ireland by 2050. Energy 2020, 207, 118264. [CrossRef] [PubMed]

13. He, G.; Mallapragada, D.S.; Bose, A.; Heuberger, C.F.; Gençer, E. Sector coupling via hydrogen to lower the cost of energy system decarbonization. arXiv 2021, arXiv:2103.03442.

14. Ihlemann, M.; Kouveliotis-Lysikatos, I.; Huang, J.; Dillon, J.; O’Dwyer, C.; Rasku, T.; Marin, M.; Poncelet, K.; Kiviluoma, J. SpineOpt: A flexible open-source energy system modelling framework. Submitted for review.

15. ENTSO-E. TYNDP 2020 Main Report; Technical Report January; ENTSO-E: Brussels Belgium, 2021. 
16. Cerniauskas, S.; Jose Chavez Junco, A.; Grube, T.; Robinius, M.; Stolten, D. Options of natural gas pipeline reassignment for Hydrogen: Cost assessment for a Germany case study. Int. J. Hydrogen Energy 2020, 45, 12095-12107. [CrossRef]

17. Schmidt, O.; Gambhir, A.; Staffell, I.; Hawkes, A.; Nelson, J.; Few, S. Future cost and performance of water electrolysis: An expert elicitation study. Int. J. Hydrogen Energy 2017, 42, 30470-30492. [CrossRef]

18. IRENA. Green Hydrogen Cost Reduction: Scaling Up Electrolysers to Meet the 1.5degC Climate Goal; Technical Report; IRENA: Abu Dhabi, United Arab Emirates, 2020.

19. SpineProject. Case Study C3 Input Data. Available online: https://github.com/Spine-project/spine-cs-c3 (accessed on 12 November 2021).

20. SpineProject. SpineOpt Repository. Available online: https://github.com/Spine-project/SpineOpt.jl (accessed on 12 November 2021).

21. Cole, W.; Frazier, A.W.; Augistine, C. Cost Projections for Utility-Scale Battery Storage: 2021 Update; Technical Report June; National Renewable Energy Laboratory: Golden, CA, USA, 2021.

22. O'Dwyer, C.; Vorushylo, I.; Huang, Y.; Hewitt, N.; Flynn, D. Enabling flexible operation of CCS plant within a high renewables power system. In Proceedings of the International Conference on Renewable Power Generation (RPG), online, 14-15 October 2021; pp. 1-6.

23. SpineProject. SpineOpt Documentation. Available online: https://spine-project.github.io/SpineOpt.jl/latest/index.html (accessed on 12 November 2021).

24. SpineProject. Spine Periods Repository. Available online: https://github.com/Spine-project/SpinePeriods.jl (accessed on 12 November 2021).

25. IBM. ILOG CPLEX Optimization Studio 12.9.0. Available online: https://www.ibm.com/docs/en/icos/12.9.0 (accessed on 12 November 2021).

26. Welder, L.; Ryberg, D.S.; Kotzur, L.; Grube, T.; Robinius, M.; Stolten, D. Spatio-temporal optimization of a future energy system for power-to-Hydrogen applications in Germany. Energy 2018, 158, 1130-1149. [CrossRef]

27. Rabiee, A.; Keane, A.; Soroudi, A. Green Hydrogen: A new flexibility source for security constrained scheduling of power systems with renewable energies. Int. J. Hydrogen Energy 2021, 46, 19270-19284. [CrossRef]

28. Helist'o, N.; Kiviluoma, J.; Morales-España, G.; O’Dwyer, C. Impact of operational details and temporal representations on investment planning in energy systems dominated by wind and solar. Appl. Energy 2021, 290, 116712. [CrossRef]

29. Poncelet, K.; Delarue, E.; Six, D.; Duerinck, J.; D’haeseleer, W. Impact of the level of temporal and operational detail in energy-system planning models. Appl. Energy 2016, 162, 631-643. [CrossRef]

30. Gonzato, S.; Bruninx, K.; Delarue, E. Long term storage in generation expansion planning models with a reduced temporal scope. Appl. Energy 2021, 298, 117168. [CrossRef]

31. Poncelet, K.; Hanspeter, H.; Delarue, E.; Virag, A.; D’haeseleer, W. Selecting representative days for capturing the implications of integrating intermittent renewables in generation expansion planning problems. IEEE Trans. Power Syst. 2017, 32, 1936-1948. [CrossRef] 